\title{
Dynamics in shear flow studied by X-ray Photon Correlation Spectroscopy
}

\author{
Sebastian Busch, ${ }^{1}$ Torben Haugaard Jensen, ${ }^{2}$ Yuriy Chushkin,${ }^{3}$ and Andrei Fluerasu ${ }^{3}$ \\ ${ }^{1}$ Physik Department, TU München, Garching bei München, Germany \\ ${ }^{2}$ Niels Bohr Institute, University of Copenhagen, Denmark \\ ${ }^{3}$ European Synchrotron Radiation Facility, Grenoble, France
}

(Dated: August 21, 2021)

\begin{abstract}
X-ray photon correlation spectroscopy was used to measure the diffusive dynamics of colloidal particles in a shear flow. The results presented here show how the intensity autocorrelation functions measure both the diffusive dynamics of the particles and their flow-induced, convective motion. However, in the limit of low flow/shear rates, it is possible to obtain the diffusive component of the dynamics, which makes the method suitable for the study of the dynamical properties of a large class of complex soft-matter and biological fluids. An important benefit of this experimental strategy over more traditional X-ray methods is the minimization of X-ray induced beam damage. While the method can be applied also for photon correlation spectroscopy in the visible domain, our analysis shows that the experimental conditions under which it is possible to measure the diffusive dynamics are easier to achieve at higher q values (with X-rays).
\end{abstract}

\section{INTRODUCTION}

X-ray photon correlation spectroscopy (XPCS) offers an unique way to perform direct measurements on the mesoscale dynamics in a large class of complex soft-

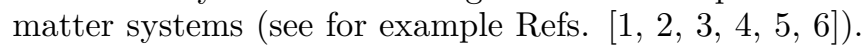
Based on the same principle as Dynamic Light Scattering (DLS) 7], XPCS is however not limited to non-turbid samples, multiple scattering can usually be neglected, and can access larger values of the momentum transfer $\vec{q}$, probing smaller spacial distances. Two major difficulties associated with XPCS are the small scattering strength of most samples and their degradation induced by the Xrays. Using a high-brilliance $3^{\text {rd }}$ generation synchrotron source helps addressing the former issue but increases at the same time the importance of the latter. In the case of fluid samples, performing XPCS experiments under continuous flow can limit the effects of beam damage. In addition, fluid mixers, in which the temporal dependence of a process taking place in a flow device is mapped into a spatial dependence along the flow channel, can be used to perform time-resolved studies [8, 9].

In the experiments reported here, XPCS was used to study the Brownian dynamics of a colloidal suspension of (weakly to non-interacting) hard spheres under shear flow. In general, the correlation functions measured by XPCS are affected by both, the flow-induced dynamics as well as the diffusive dynamics of the particles. While measuring the flow velocity profile can be a legitimate goal in many applications (see for example Ref. [10]), the aim of the present experiment was to describe the diffusive component of the dynamics. The results presented here show that for a laminar flow, and in the limits of low shear rates, it is possible to decouple the components due to diffusive and convective motion of the particles.

\section{DESCRIPTION OF THE EXPERIMENT}

\section{A. X-ray photon correlation spectroscopy}

The experiment was performed at the Troikka beamline (ID10A) of the European Synchrotron Radiation Facility (ESRF) in Grenoble, France. A single bounce $\mathrm{Si}(111)$ crystal monochromator was used to select $8 \mathrm{keV} \mathrm{X}$-rays, corresponding to a wavelength of $\lambda=1.55 \AA$. The relative bandwidth was $\Delta \lambda / \lambda \approx 10^{-4}$. A Si mirror downstream of the monochromator was suppressing higher order frequencies. The source size (FWHM) was approximately $30 \times 900 \mu \mathrm{m}^{2}(\mathrm{v} \times \mathrm{h})$ and the source-sample distance was $46 \mathrm{~m}$. In the vertical direction, the X-ray beam was focused by a beryllium compound refractive lens in order to enhance the flux. A transversely partially coherent beam was defined by slit blades with highly polished cylindrical edges. The slit size was $10 \mu \mathrm{m}$ in the vertical and horizontal directions. A set of guard slits, placed just upstream of the sample was used to minimize the parasitic X-ray scattering from the beam-defining slits (see Fig. 17). Under these conditions, the incident X-ray flux was $\sim 5 \cdot 10^{9}$ photons/s. The scattering from the colloidal suspension was recorded with a Cyberstar scintillator point detector placed at a distance of $2.30 \mathrm{~m}$ from the sample. The detection area was typically limited to $100 \times 100 \mu \mathrm{m}^{2}$ by precision slits placed in front of the detector, corresponding to a few speckles.

As it will be seen, the dynamics measured in a flowing sample is not isotropic. In the experiments described here, measurements were performed at momentum transfers covering a range of $q \cdot a$ from 1.5 to 5.7 ( $a$ is the radius of the particles) in "transverse flow" and "longitudinal flow" geometries (Fig. 1a).

Normalized intensity fluctuation autocorrelation functions,

$$
g_{2}(\vec{q}, t)=\frac{\left\langle I\left(\vec{q}, t_{0}\right) \cdot I\left(\vec{q}, t_{0}+t\right)\right\rangle_{t_{0}}}{\left\langle I\left(\vec{q}, t_{0}\right)\right\rangle_{t_{0}}^{2}}
$$




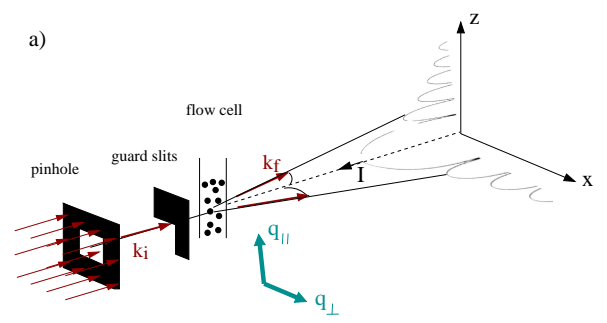

b)

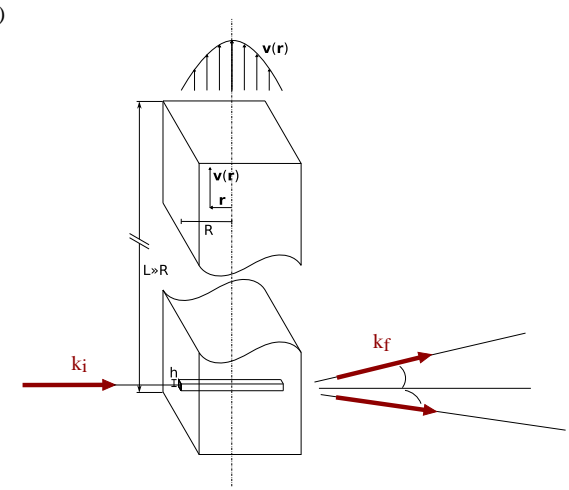

FIG. 1: (a) XPCS experimental setup for the flow geometry considered here. Longitudinal flow scans were performed by moving the detector in the vertical $z$ direction, while transverse flow scans were performed by keeping the detector in the horizontal scattering plane and moving it along the $x$ direction. (b) Schematics of the flow channel with quadratic section (edge length $2 R$ ). The velocity profile has a parabolic shape; $h$ is the transverse size of the X-ray beam.

were obtained using a "Flex" hardware correlator manufactured by Correlator.com that was connected to the output of the X-ray detector.

\section{B. Flowcell}

The in-house made flowcell consisted of an aluminum sheet through which the flow channel (see sketch in Fig. 1b) was cut by high-precision machining. Polymercoated kapton foils were fusion-bonded to the face of the aluminum and held in place by a "sandwich-type" pressure applying system. A high-precision syringe pump obtained from Harvard Apparatus was used to push the fluid through the flow device. The width of the channel $(2 R=1000 \mu \mathrm{m})$ was much larger than the one of the beam ( $h=10 \mu \mathrm{m})$. Therefore, the scattering volume could be approximated by a one-dimensional object. This made it possible to apply the model of a Poiseuille flow - a Newtonian fluid performing a shear flow in a cylindrically-shaped flow device.

The laminar character of the flow is determined by the Reynolds number which measures the ratio of the inertial to viscous forces, and is defined as $R e=\frac{v_{0} 2 R \rho}{\eta}[9]$. Here $2 R$ is the characteristic length of the system (confer to Fig. 1 b), $v_{0}$ is the flow velocity of the fluid and $\eta$ the dynamic viscosity. As long as the Reynolds numbers are

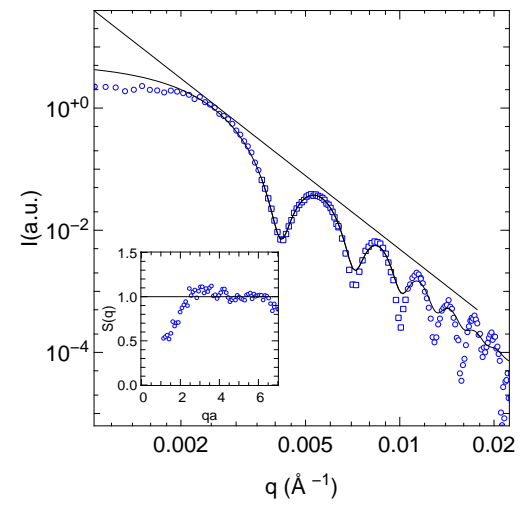

FIG. 2: Static SAXS scattering from the latex particles and fitted form factor for the solid spherical particles (solid line). The straight line with slope -4 , representing Porod's law is a guide for the eyes. Inset: estimate of the structure factor $S(q)$ obtained by dividing the total scattered intensity to the fitted value for the form factor.

low $\left(R e \ll 10^{3}\right)$, the flow is in a laminar regime, which was well the case in all the measurements performed here, as can be seen in table I. Assuming a no-slip boundary condition at the wall of the flow cell, the velocity profile can therefore be described by

$$
v(r)=2 v_{0}\left(1-\frac{r^{2}}{R^{2}}\right) .
$$

Here (see Fig. 1b), $r$ is the distance from the center of the capillary and $v_{0}$ is the average flow velocity (defined using the average flow rate $\left.Q=4 R^{2} v_{0}\right)$. The mean shear rate is defined as $\dot{\gamma}=\frac{2 v_{0}}{R}$.

\section{Sample}

Charge stabilized latex spheres with a nominal radius of $a=110 \mathrm{~nm}$ dispersed in water were obtained from Duke Scientific Co. at a concentration of $10 \%$ per weight. Glycerol with a purity of $\geq 99 \%$ was obtained from SigmaAldrich Co. The spheres suspension was mixed with the glycerol and placed in a dessicator which was evacuated on top of a heatable magnetic stirrer. The temperature was set to approximately $75^{\circ} \mathrm{C}$ which facilitated water evaporation and decreased the viscosity. These conditions were applied for about two days, leaving the samples not only essentially water-free but also de-gased, avoiding thus an important reason for bubble formation. The amount of suspension and glycerol was chosen such that the final volume fraction was $\Phi=0.1$.

The small angle X-ray scattering (SAXS) pattern of the sample is shown in Fig. 2. The time-averaged data was fitted, for values of $q a \geq 5.0$, to the form factor of spherical particles with the size distribution given by a Schultz distribution function, yielding a radius of $a=$ $1070 \AA$ and a size polydispersity $\leq 7 \%$. Also shown is 
the static structure factor $S(q)$, exhibiting the weakness of interactions $(S(q) \approx 1$ for most of the $q$-range).

\section{THEORY: XPCS IN A LAMINAR FLOW}

The central quantity describing the dynamics of the colloidal suspension is the dynamic structure factor, or intermediate scattering function (ISF), which in the case of non-interacting (statistically independent) particles is given by [7]

$g_{1}(\vec{q}, t) \propto \sum_{k=1}^{N}\left\langle E_{k}^{*}(0) E_{k}(t) \exp \left[-i \vec{q} \cdot\left({\overrightarrow{r^{\prime}}}_{k}(0)-{\overrightarrow{r^{\prime}}}_{k}(t)\right)\right]\right\rangle$.

Here $\mathbf{q}$ is the scattering vector, $E_{k}(t)$ is the amplitude of X-rays scattered by particle $k$ at time $\mathrm{t}$ and ${\overrightarrow{r^{\prime}}}_{k}(t)$ is the particle position in the presence of flow at time $t$. The summation is over all particles in the scattering volume for a particular realization of the experiment and the ensemble average is performed over many different realizations. In order to differentiate between the diffusive and the flow-induced particle displacements, it is useful to write

$$
\begin{aligned}
& \exp \left[-i \vec{q} \cdot\left({\overrightarrow{r^{\prime}}}_{k}(0)-{\overrightarrow{r^{\prime}}}_{k}(t)\right)\right]= \\
& \exp \left[-i \vec{q} \cdot\left(\vec{r}_{k}(0)-\vec{r}_{k}(t)\right)\right] \cdot \exp \left(i \vec{q} \cdot \vec{v}_{0} t\right) \cdot \exp (i \vec{q} \cdot \delta \vec{v} t) .
\end{aligned}
$$

Here $\vec{r}_{k}(t)$ is the particle position due to only to the diffusive motion, $\vec{v}_{0}$ is the average flow velocity throughout the sample and $\delta \vec{v}$ is the flow velocity difference between positions $\vec{r}_{k}(t)$ and $\vec{r}_{k}(0)$.

By analyzing Eqs. 3] and 4, it can be concluded that the ISF depends on three main factors:

i) the particle diffusion, with a characteristic time scale $\tau_{D}=\left(D q^{2}\right)^{-1}(D$ is the diffusion coefficient, see below $)$

ii) the amplitude factor, which measures the intensity scattered by each particle, $I_{k}=\left\langle E_{k}^{*}(0) \cdot E_{k}(0)\right\rangle$, but also the transit time of the particles through the scattering volume, $\tau_{T}=h / v_{0}$ ( $h$ is the transverse beam size $)$,

iii) shear-induced effects which are described, as it will be shown, by a time scale $\tau_{S} \propto(q \dot{\gamma} R \cos \phi)^{-1}$, which is inversely proportional to the magnitude of the local velocity gradient $\dot{\gamma}$. Here $\phi$ is the angle between the scattering vector and the local velocity $\vec{v}$.

Note that the importance of the fourth factor, namely the average velocity-dependent $\exp \left[i \vec{q} \cdot \vec{v}_{0} t\right]$, was not emphasized because it can only be detected in heterodyne correlation functions 11]. A homodyne PCS experiment (like the one reported here) detecting the square modulus of the ISF, $\left|g_{1}(\mathbf{q}, t)\right|^{2}$ can measure velocity gradients but not absolute velocities [12].

It can be demonstrated [13] that, if the three time scales described above are sufficiently different from each other, the ISF can be factorized in independent contributions from the three relaxation mechanisms. The physical argument is that if one of the contributions (e.g. diffusion) to the ISF varies on a much faster time scale than the others, then the particles explore the phase space associated with this particular relaxation mechanism while the other factors are (approximately) constant. In such a situation, the ISF under shear flow can be written as

$$
\begin{aligned}
g_{1}(\vec{q}, t) \propto & \exp \left(i \vec{q} \vec{v}_{0} t\right)\left\langle E^{*}(0) E(t)\right\rangle \times \\
& \left\langle\sum_{k=1}^{N} \exp \left[-i \vec{q} \cdot\left(\vec{r}_{k}(0)-\vec{r}_{k}(t)\right)\right]\right\rangle \times \\
& \left\langle\sum_{k, l=1}^{N} \exp [i \vec{q} \cdot \delta \vec{v}(k, l) t]\right\rangle .
\end{aligned}
$$

In writing Eq. 5, we have also assumed that all particles are identical scatterers and that, due to the fact that particles are indistinguishable and that the system is stationary, the shear induced effects can be calculated also by taking into account the velocity differences between all pair of particles for a particular realization of the statistical ensemble.

The physical interpretation of the shear-induced effects is that the frequencies of X-rays scattered by particles which are moving with different (average) velocities are Doppler-shifted with respect to each other, and produce a beat frequency when interfering on the detector. For a pair of particles situated at two different locations and moving with a velocity difference of $\delta \vec{v}$, the self-beat frequency induced by the Doppler shifts is $\vec{q} \cdot \delta \vec{v}[7,10,13$. From here, it can be seen that measurements with $\vec{q} \| \vec{v}$ will be influenced by these velocity-differences while measurements with $\vec{q} \perp \vec{v}$ are not affected because the scalar products $\vec{q} \cdot \overrightarrow{\delta v}$ are all zero.

In the case of practical interest for our study, namely the situation in which the diffusion relaxation time is much shorter than the other relevant time scales, it is natural to assume that the scattered intensity fluctuations are well described by Gaussian statistics and that the intensity autocorrelation functions are related to the intermediate scattering functions by the Siegert relationship,

$$
g_{2}(\vec{q}, t)=1+\beta \cdot\left|g_{1}(\vec{q}, t)\right|^{2},
$$

where $\beta$ is the speckle contrast which was, in the described experimental setup, of the order of $5 \%$. In ref. 13] it is also mentioned that "it can be shown" that this relation is also valid for the case where the flow-induced deterministic motions dominate those due to diffusion, provided that the decay of the ISF is sufficiently short compared to the inverse local velocity gradient $\dot{\gamma}^{-1}$. This condition is almost always fulfilled with typical experimental parameters, and in particular is satisfied for our longitudinal flow scattering geometry $(\vec{q} \| \vec{v})$, which justifies the use of the Siegert relationship in all the experiments performed here.

In conclusion, the homodyne correlation functions measured in our XPCS experiments can be well described 
by,

$$
\left|g_{1}(\vec{q}, t)\right|^{2}=\left|g_{1, D}(\vec{q}, t)\right|^{2} \cdot\left|g_{1, T}(t)\right|^{2} \cdot\left|g_{1, S}(\vec{q}, t)\right|^{2},
$$

where the first factor (subscript $D$ ) is due to thermal diffusion, the second one (subscript $T_{T}$ ) to the transit time through the scattering volume, and the last one (subscript $S$ ) is due to shear. Each of these factors are discussed in the following.

\section{A. Thermal diffusion}

The intermediate scattering function for a non-flowing sample undergoing Brownian motion is a simple exponential decay with the relaxation rate determined by the diffusion coefficient $D$ and scattering wavevector $q$,

$$
\left|g_{1, D}(\vec{q}, t)\right|^{2}=\exp \left[-2 D q^{2} t\right] .
$$

In this case, the dynamics is isotropic and does not depend on the direction of $\mathbf{q}$, but only on its absolute value $q$. When a uniform shear rate $\dot{\gamma}$ is applied, the diffusion is in general enhanced by the shear and becomes anisotropic, changing the formula to [14]:

$\left|g_{1, D}(\vec{q}, t)\right|^{2}=\exp \left[-2 D q^{2} t\left(1-\frac{q_{\|} q_{\perp}}{q^{2}} \dot{\gamma} t+\frac{1}{3} \frac{q_{\|}^{2}}{q^{2}}(\dot{\gamma} t)^{2}\right)\right]$.

Here, $q_{\|}$and $q_{\perp}$ are the components of $\vec{q}$ parallel and respectively perpendicular to the direction of the flow. In a transverse scattering geometry $(\vec{q} \perp \vec{v}), q_{\|}=0$ and Eq. (9) reduces to Eq. (8). This is obviously not true when $\vec{q} \| \vec{v}$, in which case the diffusion is enhanced by the shear. However, at the shear rates used here (see table【), the products $t \dot{\gamma}$ are of the order of $10^{-3}$ and Eq. (9) provides only a minor correction to Eq. (8), which can still be considered to describe well the thermal diffusion of the colloids.

\section{B. Transit-time effects}

When the colloidal suspension flows through the beam, new particles enter the scattering volume, replacing particles that leave on the other side. The "refilling" of a scattering volume of transverse size $h$, leads to a loss of correlation of the dynamic structure factor that can be characterized by a frequency $\nu_{\text {tr }} \propto v_{0} / h$.

The Deborah-number is a measure for the importance of this effect compared to the decorrelation due to thermal diffusion taking place on a timescale $\left(D q^{2}\right)^{-1}$. In this context, the Deborah number can be defined as,

$$
D e=v_{0}\left(h D q^{2}\right)^{-1} .
$$

Typical values for two values of $q$ are given in table 1

It was demonstrated [7, 15, 16, 17] that the contribution of the transit effect to the correlation function

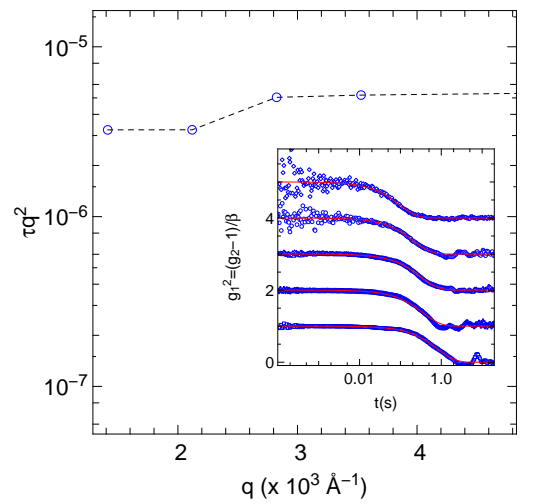

FIG. 3: Correlation functions taken at no flow, $Q=0$ (sample in a capillary) at all values of the scattering wavevector measured, $q=1.8,2.1,2.8,2.5$, and $5.3 \times 10^{-3} \AA^{-1}$. The inset shows the normalized correlation functions shifted vertically, for clarity, by $0,1,2,3$, and respectively 4 , and fits with exponential decays - Eq. (8), solid lines. The main graph shows the correlation times $\tau=1 / D q^{2}$ (multiplied by $q^{2}$ in order to eliminate the q-dependence) as a function of $q$.

is reflecting the beam profile, being "scanned" by the flowing particles. As the beam is defined by rectangular slits, the incident intensity follows a $\left(\frac{\sin (r)}{r}\right)^{2}$ distribution, the squared Fourier transform of a rectanglefunction. Due to the guard slits, which are aligned to suppress the higher order peaks, the beam profile at the sample position can be well approximated by a Gaussian form, leading to

$$
\left|g_{1, T}(t)\right|^{2} \propto \exp \left[-\left(\nu_{\mathrm{tr}} t\right)^{2}\right] .
$$

\section{Shear-induced effects}

The shear-induced contribution to the correlation function, $\left|g_{1, S}(\vec{q}, t)\right|^{2}$ can be written as a sum over all pairs of particles or as a double integral over the scattering volume which can be approximated by a line of length $2 R$,

$$
\left|g_{1, S}(\vec{q}, t)\right|^{2}=\frac{1}{4 R^{2}} \int_{-R}^{R} \int_{-R}^{R} \cos \left(\vec{q} t \overrightarrow{\delta v}\left(r_{1}, r_{2}\right)\right) \mathrm{d} r_{1} \mathrm{~d} r_{2} .
$$

The integral can be performed analytically for a uniform shear rate (Couette geometry) [10], leading to

$$
\left|g_{1, S}(\vec{q}, t)\right|^{2}=\frac{\sin \left(q_{\|} v_{0} t\right)}{q_{\|} v_{0} t} .
$$

In Appendix A, Eq. (12) was also solved analytically for a different flow geometry, a Poiseuille flow described by a parabolic velocity profile Eq. (2), which provides a better description for the data presented here. The resulting shear-induced response to the correlation functions can be written using the error function of a complex 
argument,

$$
\left|g_{1, S}(\vec{q}, t)\right|^{2}=\frac{\pi^{2}}{16 q_{\|} t v_{0}}\left|\operatorname{erf}\left(\sqrt{\frac{4 i q_{\|} t v_{0}}{\pi}}\right)\right|^{2} .
$$

From Eq. (13) and (14), a shear-induced frequency can be defined as

$$
\Gamma_{S}=\mathbf{v}_{\mathbf{0}} \cdot \mathbf{q}
$$

If the scattering alignment is supposed to be perfectly transversal, $\mathbf{q} \perp \mathbf{v}, \Gamma_{S}=0$ and the XPCS correlation functions are unaffected by the shear-induced effects.

The relative importance of the shear-induced effects compared to thermal diffusion is usually described by the Peclet number $P=\frac{\dot{\gamma} R^{2}}{D}$. As it can be seen in table【 the Peclet numbers are very high even at low shear rates, and the shear-induced effects are the dominating contribution to the measured correlation functions if a non-transverse scattering geometry is used.

Following the discussion above, it turns out that the relative influence of the shear can also be expressed in terms of a different dimensionless number which will be called here Shear number, given by the ratio between the characteristic diffusion time $\tau_{D}=1 / D q^{2}$ and the sheartime, $\tau_{S}=1 / \Gamma_{S}=1 / \mathbf{q} \cdot \mathbf{v}_{\mathbf{0}}$,

$$
S=\frac{\mathbf{q} \cdot \mathbf{v}_{\mathbf{0}}}{D q^{2}} \text {. }
$$

Values for the shear number $S$ are given in table I for a value of the longitudinal component of the scattering wavevector $q_{\|}=1.4 \cdot 10^{-3} \AA^{-1}$. The conclusion is the same as the one resulting from the evaluation of the Peclet numbers - the shear induced effects are the dominating ones if a non-transverse scattering geometry is used and it is hard or impossible to measure the thermal diffusion of the particles in such a scattering geometry.

With the three components of the correlation functions determined by thermal diffusion, shear, and transittime described by Eqs. (9), (14), and respectively (11), the normalized intensity fluctuation autocorrelation functions measured in a XPCS experiment with a sample undergoing shear flow can be written as,

$$
\begin{aligned}
& \left|g_{1}(\mathbf{q}, t)\right|^{2}=\left(g_{2}(\mathbf{q}, t)-1\right) / \beta= \\
& \quad \exp \left[-2 D q^{2} t\left(1-\frac{q_{\perp} q_{\|}}{q^{2}} \dot{\gamma} t+\frac{q_{\|}^{2}}{q^{2}} \frac{(\dot{\gamma} t)^{2}}{3}\right)\right] . \\
& \quad \exp \left[-\left(\nu_{\mathrm{tr}} t\right)^{2}\right] \cdot \frac{\pi^{2}}{16 q_{\|} t v_{0}}\left|\operatorname{erf}\left(\sqrt{\frac{4 i q_{\|} t v_{0}}{\pi}}\right)\right|^{2} .
\end{aligned}
$$

As it will be shown in the following section, Eq. (17) fits very well the measured correlation functions (Fig. (4)

\section{RESULTS \& DISCUSSION}

In the $q$-range probed here, the static structure factor (Fig,2, inset) of the colloidal suspension in glycerol
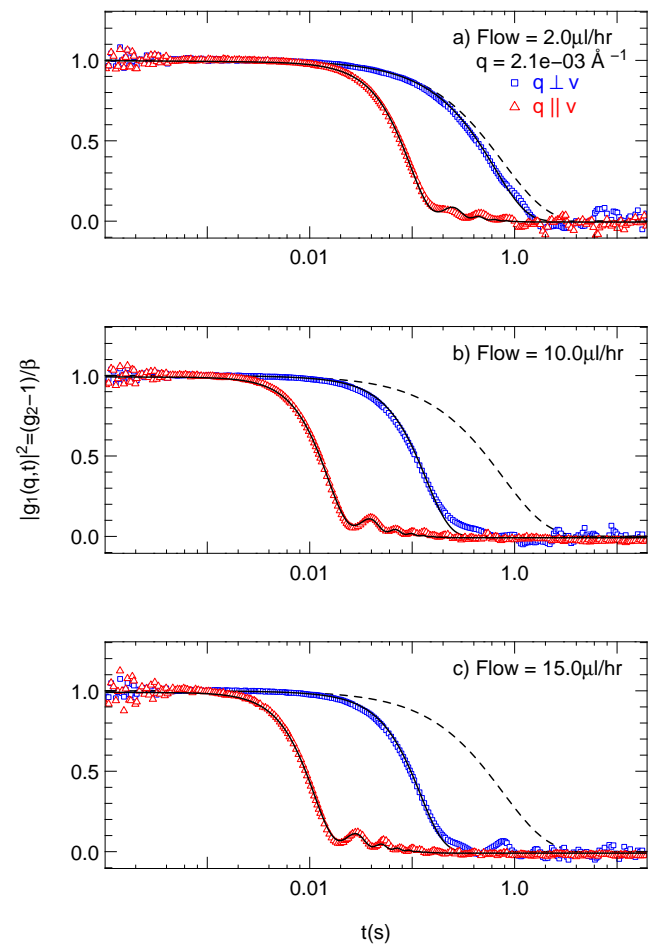

FIG. 4: Normalized correlation functions for a single wavevector, $q=2.1 \cdot 10^{-3} \AA^{-1}$ and three different flow rates: a) $Q=2$, b) $Q=10$, and c) $Q=15 \mu \mathrm{l} / \mathrm{h}$. The dashed line shows the fit of a simple exponential to the zero-flow (reference) correlation function for the same value of $q$. The solid lines show fits with (19) and (20) as described in the text.

exposed to a shear flow is isotropic and independent of the flow rate. This means that the flow does not change the spacial probability distribution of the particles. However, the dynamic structure factor (intermediate scattering function) is both flow-dependent and anisotropic.

Fitting the data obtained from a non-flowing sample with a simple exponential decay - Eq. (8), as shown in Fig. 3 (inset), yields a mean diffusion coefficient of $D=$ $2.39 \cdot 10^{-15} \frac{\mathrm{m}^{2}}{\mathrm{~s}}$. Using the Stokes-Einstein relation

$$
D=\frac{k_{B} T}{6 \pi \eta a},
$$

where $k_{B}$ is the Boltzmann constant and $T=293 \mathrm{~K}$ the temperature, the dynamic viscosity $\eta$ can be calculated to be $0.839 \mathrm{~Pa} \cdot \mathrm{s}$ which corresponds to a left-over water content of $3 \%$ [18].

Using the obtained diffusion coefficient and viscosity, the flow can be completely characterized (see relevant values in table I). It can be concluded that the Reynoldsnumbers are several orders of magnitude below the onset of turbulent flow. The Deborah-number - Eq. (10) is $q$ dependent. At the smallest $q$-values measured, it is not possible any more to assume $D e \ll 1$ (even at low flow rates) and the transit-time effects must be considered for an accurate description of the correlation functions. At higher values of $q$, the Deborah numbers $D e$ become 
TABLE I: Characterizing quantities of the flow of the sample. As a function of flow rate $Q$, the mean velocity $v_{0}$, Reynoldsnumber $R e$, Deborah-number $D e$ (for two $q$-values), shear rate $\dot{\gamma}$, Peclet number $P$, and Shear number (16) are shown.

\begin{tabular}{l|rrrrrrrrr}
\hline$Q[\mu \mathrm{l} / \mathrm{h}]$ & 0.0 & 2.0 & 5.0 & 7.5 & 10 & 15 & 20 & 40 & 80 \\
\hline$v_{0}[\mu \mathrm{m} / \mathrm{s}]$ & 0.0 & 0.5 & 1.3 & 2.0 & 2.7 & 4.1 & 5.5 & 11 & 22 \\
$R e\left[10^{-6}\right]$ & 0.0 & 1.7 & 4.2 & 6.3 & 8.4 & 13 & 17 & 33 & 67 \\
$D e\left(q=1.4 \cdot 10^{-3} \AA^{-1}\right)$ & 0.0 & 0.12 & 0.30 & 0.44 & 0.59 & 0.89 & 1.2 & 2.4 & 4.7 \\
$D e\left(q=5.3 \cdot 10^{-3} \AA^{-1}\right)$ & 0.0 & 0.0083 & 0.021 & 0.031 & 0.041 & 0.062 & 0.083 & 0.17 & 0.33 \\
$\dot{\gamma}\left[\frac{10^{-3}}{s}\right]$ & 0.0 & 1.1 & 2.8 & 4.2 & 5.6 & 8.3 & 11 & 22 & 44 \\
$P\left[10^{6}\right]$ & 0.0 & 0.46 & 1.2 & 1.7 & 2.3 & 3.5 & 4.6 & 9.3 & 19 \\
$S\left(q_{\|}=1.4 \cdot 10^{-3} \AA^{-1}\right)$ & 0.0 & 36 & 93 & 143 & 193 & 293 & 393 & 786 & 1429 \\
\hline
\end{tabular}

much smaller and the transit-time effects are less important. Increasing the beam size $h$ offers another practical possibility to lower the Deborah number, if working with a lower speckle contrast is acceptable. The Peclet number $P$ and/or the q-dependent shear numbers $\mathrm{S}$ are much larger than unity even at the smallest flow rates, showing that the shear-induced effects are always important.

The intensity fluctuation correlation functions were measured for several values of the scattering wavevector $\mathbf{q}$ in both transverse $(\vec{q} \perp \vec{v})$ and longitudinal $(\vec{q} \| \vec{v})$ scattering geometries and for several flow rates between 0 and $80 \mu \mathrm{l} / \mathrm{h}$ and fitted using Eq. (17). For a transverse scattering geometry $q_{\|}=0$, and Eq. (17) leads to

$$
g_{2, \perp}(q, t)=1+\beta \cdot \exp \left[-2 \frac{t}{\tau}\right] \cdot \exp \left[-\left(\nu_{\mathrm{tr}} t\right)^{2}\right]
$$

with the diffusion time $\tau=1 / D q^{2}$ and the transitinduced frequency $\nu_{t r}$ as fitting parameters (in addition to the speckle contrast $\beta$ and the baseline value). The diffusion coefficient was obtained by fitting the low-flow transverse, $\vec{q} \perp \vec{v}$ correlation functions and was subsequently fixed in the high-flow data which were used to determine the flow-dependent, transit-induced frequency $\nu_{\operatorname{tr}}$. Both parameters $\tau$ and $\nu_{\operatorname{tr}}$ were fixed to the values obtained from transverse scans when fitting the $\vec{q} \| \vec{v}$ data with

$$
\begin{aligned}
g_{2, \|}(q, t)=1+ & \beta \cdot \exp \left[-2 \frac{t}{\tau}\left(1+\frac{(\dot{\gamma} t)^{2}}{3}\right)\right] \\
& \cdot \exp \left[-\left(\nu_{\mathrm{tr}} t\right)^{2}\right] \cdot \frac{\pi\left|\operatorname{erf}\left(\sqrt{i \Gamma_{S} t}\right)\right|^{2}}{4 \Gamma_{S} t},
\end{aligned}
$$

from which a fitted shear-induced frequency $\Gamma_{S}$ was obtained.

Example fits for a single value of $q$ and three different flow rates are shown in Fig. 4. The parameters resulting from the fitting procedure described above, $\nu_{\mathrm{tr}}$ and $\Gamma_{S}$, are plotted in Fig. 5 as a function of the nominal flow velocity $v_{0}$ computed from the volume flow rate. The shear rate $\dot{\gamma}$ was left in place in Eq. (20) only for reasons of logical consistency with Eq.(17) and (9), but its influence on the shape of the correlation functions is too small (for any reasonable values of the shear rate) to allow any meaningful fitting. As it can be seen from Fig. 4.
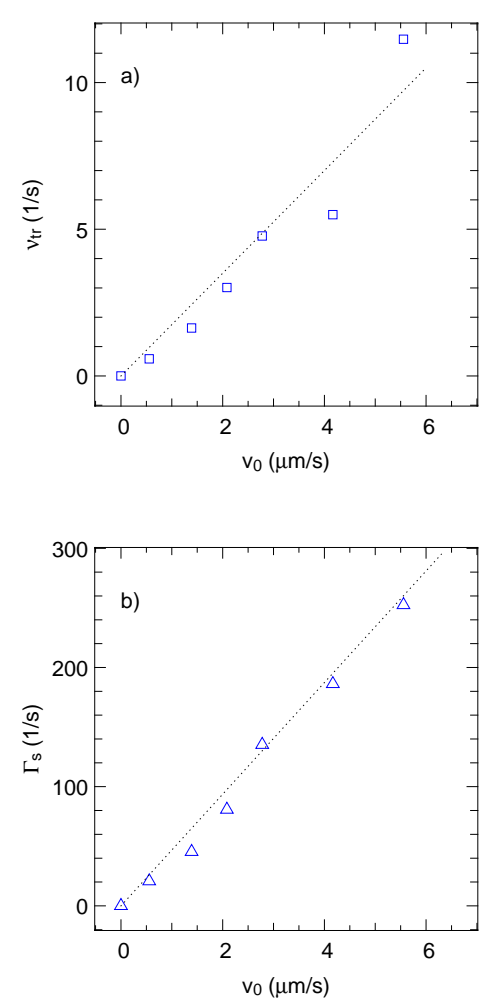

FIG. 5: The fitted parameters versus the flow velocity of the sample; a) shows the results of fitting the transverse correlation functions with Eq. (19). Fits with Eq. (20) of the longitudinal correlation functions yields the values for the shearinduced frequency $\Gamma_{S}$ displayed in b).

the quality of the fits is quite remarkable, especially for $\vec{q} \| \vec{v}$ and for the higher flow rates, even if the only dominant parameter for these fits is $\Gamma_{S}$, the shear-induced frequency.

From a practical point of view, in order to be able to measure the diffusive component of the particle dynamics under flow, its relative importance must be high compared with that of the shear and transit-time induced effects. Keeping a transverse scattering geometry is the only method to limit the shear-induced effects, but in practice any small misalignment becomes the limiting factor at high-enough flow rates. If $\phi$ is the angle between 


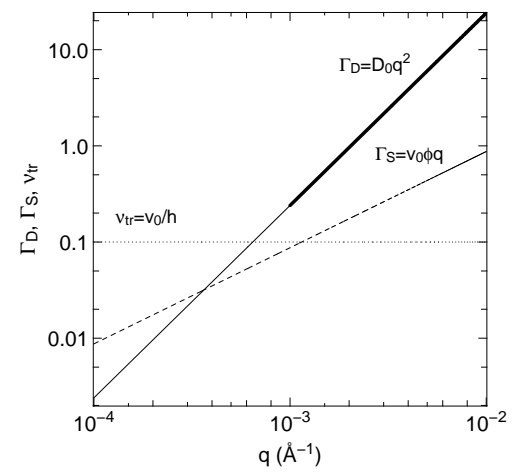

FIG. 6: Dispersion relationships for the diffusion $\left(\Gamma_{D}=D q^{2}\right)$, shear $\left(\Gamma_{S}=v_{0} \phi q\right)$, and transit $\left(\nu_{t r}=v_{0} / h\right)$ relaxation rates. The (example) values used here to estimate $\Gamma_{D}, \Gamma_{S}$, and $\nu_{t r}$ were $D_{0} \approx 2.4 \cdot 10^{5} \AA / \mathrm{s}, \phi \approx 0.01(0.5 \mathrm{deg}), v_{0}=1 \mu \mathrm{m} / \mathrm{s}$. The thick solid line shows the q region where the thermal diffusion dominates the dynamic signal and can be measured by XPCS.

$\mathbf{q}$ and the direction orthogonal to $\mathbf{v}_{\mathbf{0}}$, the shear-induced relaxation rate - Eq.(15) - becomes [19],

$$
\Gamma_{S}=v_{0} \phi q
$$

To illustrate the impact of even small misalignments, the dispersion relationships for thermal diffusion $\Gamma_{D}=$ $D q^{2}$, shear-induced effects, Eq.(21), with $\phi$ assumed to be 0.01 (corresponding to a misalignment of $0.5 \mathrm{deg}$ ) and $v_{0}=1 \mu \mathrm{m} / \mathrm{s}$, are plotted in Fig. 6 together with the (qindependent) dispersion for the transit-time effects. In order to be able to measure the thermal diffusion of the particles, $\Gamma_{D}$ must dominate over the other, flow-induced relaxation rates. For the values chosen in this example, this condition is fulfilled in the q-range for which the diffusion dispersion relationship is highlighted. From Fig. 6 it is clear that for any flow velocity larger than a few $\mu \mathrm{m} / \mathrm{s}$, the shear-induced relaxation rates $\Gamma_{S}$ will shift upwards which would "push" the possible q-range to higher values. Equivalently, working at a fixed value of $q$ in order to obtain specific information about the dynamic properties on a certain length-scale puts a condition on the maximum flow rate for which the correlation functions are still dominated by their diffusive components. At the same time, it is also clear that in the case of light-PCS, with smaller values of $q$, these conditions are harder to fulfill 14.

From this analysis, it results that fitting the correlation functions obtained in transverse scans with Eq. (19) is justified only at the lowest flow rates probed here (assuming that it is difficult to achieve a vertical alignment better than $\phi=0.01$ ). For such low flow rates, the correlation functions will not depend much on neither the finite transit-time nor on the shear time and fits with a simple exponential form will render the correct diffusion time and/or diffusion coefficient.

The correlation times in Fig. 7 were obtained by performing fits to the transverse correlation functions with simple exponential forms. While at most of the higher

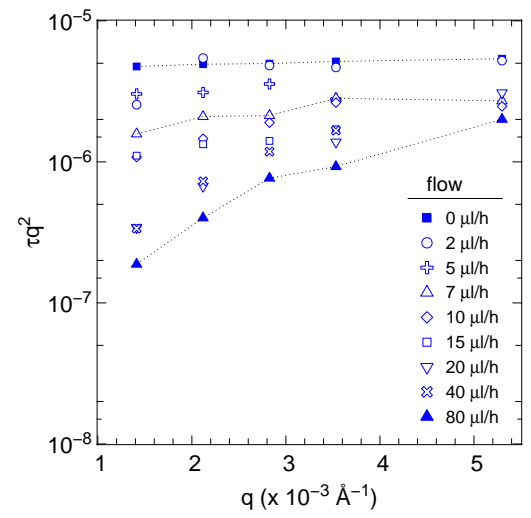

FIG. 7: Correlation times (multiplied by $q^{2}$ ) as a function of $q$. At low flow rates the correlation times are unaffected by the transit time and/or the shear and the diffusive dynamics of the particles can be measured in a flowing solution.

flow rates probed here the influence of the flow on the correlation times is clear, at the low-enough flow rates $(\mathrm{Q} \leq 2 \mu \mathrm{l} / \mathrm{h})$, the correlation times are still flowindependent and follow the $\Gamma \propto q^{2}$ dispersion relationships expected for Brownian motion.

Therefore, using XPCS on a flowing sample seems applicable to measure the "intrinsic" dynamics of the sample. While the scattering functions recorded with $\vec{q} \| \vec{v}$ are completely dominated by shear effects, these do not contribute (or contribute much less) to the spectra at $\vec{q} \perp \vec{v}$.

\section{SUMMARY}

The correlation functions from a colloidal suspension undergoing shear flow were measured by homodyne XPCS. The data could be explained by computing the intermediate scattering function as a product of the scattering functions due to diffusion, transit, and shear.

Our measurements suggest that it should be possible to measure samples under flow and obtain the diffusion coefficient and/or particle size information, etc. For shear and Deborah-numbers $<0.1$, the correction is negligible and equation (8) can be used. For higher Deborah and/or shear numbers, a more complicated convolution between different dynamic mechanisms takes place and the correlation functions are dependent on at least three important factors - thermal diffusion, transit-time, and shear. Measurements at shear and/or Deborah numbers above $\approx 1$ are completely dominated by shear-induced or (in some particular cases) transit time effects and not suitable any more for a precise extraction of diffusive properties. However, as both the $D e$ and $S$ numbers are $q$-dependent, it is possible to tune the settings within some limits to access also higher flow rates.

In practice, for a specific alignment a "calibration" procedure (i.e. probing the dynamics of a colloidal suspension as it is done here), should provide a graph such as 
the one shown in Fig. 6, which provides a lower limit for $q$ accessible for a given flow rate or, equivalently, the maximum flow rate that can be set while still being able to measure the diffusive dynamics at a specific value of $q$ with the desired degree of accuracy.

This method can therefore be used to measure the dynamical properties of many soft and biological samples which suffer from beam damage, or to perform timeresolved studies in mixing devices.

\section{ACKNOWLEDGMENTS}

We acknowledge many useful discussions with Anders Madsen, Abdellatif Moussaïd, Federico Zontone, Chiara Caronna, Jean-Baptiste Salmon, Fanny Destremaut, and help in designing the experiment and building the flowcells from Henry Gleyzolle. The work of SB and THJ was part of the student research internship program at the ESRF. They wish to acknowledge financial support from the ESRF and administrative support and guidance from Catherine Stuck and the HR department and from the ID10A staff.

\section{APPENDIX A: SHEAR-INDUCED CORRELATION}

In the following, Eq. (12) is solved for a Poiseuille flow, with a velocity profile described by (2), and a closed-form expression for the shear-induced correlation $\left|g_{1, S}(\vec{q}, t)\right|^{2}$ is calculated.

The velocity difference between two particles located at different positions across the flow channel $r_{1}$ and $r_{2}$ can be written as $\overrightarrow{\delta v}\left(r_{1}, r_{2}\right)=2 \overrightarrow{v_{0}} \frac{r_{1}^{2}-r_{2}^{2}}{R^{2}}$, the integrals over the two independent variables $r_{1}$ and $r_{2}$ are equivalent and, after applying trigonometric addition identities, the double integral in equation (12) can be reduced to the integration over a single variable $r$,

$$
\begin{aligned}
\left|g_{1, S}(\vec{q}, t)\right|^{2}= & \frac{1}{4 R^{2}}\left[\int_{-R}^{R} \mathrm{~d} r \cos \left(2 q_{\|} t v_{0} \frac{r^{2}}{R^{2}}\right)\right]^{2}+ \\
& \frac{1}{4 R^{2}}\left[\int_{-R}^{R} \mathrm{~d} r \sin \left(2 q_{\|} t v_{0} \frac{r^{2}}{R^{2}}\right)\right]^{2}
\end{aligned}
$$

These integrals can be reduced to Fresnel integrals [20],

$\left|g_{1, S}(\vec{q}, t)\right|^{2}=\frac{\pi}{4 q_{\|} t v_{0}}\left[C^{2}\left(\sqrt{\frac{4 q_{\|} t v_{0}}{\pi}}\right)+S^{2}\left(\sqrt{\frac{4 q_{\|} t v_{0}}{\pi}}\right)\right]$,

which are related by (using the imaginary number $i=\sqrt{-1}$ )

$$
C(v)+i S(v)=\frac{1+i}{2} \operatorname{erf}\left(\frac{\sqrt{\pi}}{2}(1-i) v\right)
$$

to the error-function,

$$
\operatorname{erf}(u)=\frac{2}{\sqrt{\pi}} \int_{0}^{u} e^{-t^{2}} \mathrm{~d} t
$$

A normalized expression for the shear-induced correlation function $\left|g_{1, S}(q, t)\right|^{2}$ is, therefore given by

$$
\left|g_{1, S}(\vec{q}, t)\right|^{2}=\frac{\pi^{2}}{16 q_{\|} t v_{0}}\left|\operatorname{erf}\left(\sqrt{\frac{4 i q_{\|} t v_{0}}{\pi}}\right)\right|^{2}
$$

[1] A. Fluerasu, A. Moussaïd, A. Madsen, A. Schoffield, Phys. Rev. E (R) 76, 010401(R) (2007)

[2] P. Falus, M. Borthwick, S. Narayanan, A.R. Sandy, S.G.J. Mochrie, Phys. Rev. Lett. 97, 066102 (2006)

[3] R. Bandyopadhyay, D. Liang, H. Yardimci, D.A. Sessoms, M.A. Borthwick, S.G.J. Mochrie, J.L. Harden, R.L. Leheny, Phys. Rev. Lett. 93, 228302 (2004)

[4] L.B. Lurio et al., Phys. Rev. Lett. 84, 785 (2000)

[5] D. Lumma et al., Phys. Rev. E 62, 8258 (2000)

[6] A.J. Banchio, J. Gapinski, A. Patkowski, W. Häußler, A. Fluerasu, S. Sacanna, P. Holmqvist, G. Meier, M.P. Lettinga, G. Nägele, Phys. Rev. Lett. 96, 138303 (2006)

[7] B. Berne, R. Pecora, Dynamic Light Scattering (Dover, New York, 2000)

[8] L. Pollack, M.W. Tate, A.C. Finnefrock, C. Kalidas, S. Trotter, N.C. Darnton, L. Lurio, R.H. Austin, C.A. Batt, S.M. Gruner et al., Phys. Rev. Lett. 86, 4962 (2001)

[9] J. Atencia, D.J. Beebe, Nature 437, 648 (2005)

[10] T. Narayanan, C. Cheung, P. Tong, W. Goldburg, X.L. Wu, Appl. Opt. 36, 7639 (1997)

[11] F. Livet, F. Bley, F. Ehrburger-Dolle, I. Morfin,
E. Geissler, M. Sutton, J. Synchrotron Rad. 13, 453 (2006)

[12] J.B. Salmon, S. Manneville, A. Colin, B. Pouligny, Eur. Phys. J. AP 22, 143 (2003)

[13] G.G. Fuller, J.M. Rallison, R.L. Schmidt, L.G. Leal, J. Fluid Mech. 100, 555 (1980)

[14] B.J. Ackerson, N.A. Clark, J. Physique 42, 929 (1981)

[15] R.V. Edwards, J.C. Angus, M.J. French, J. John W. Dunning, Journal of Applied Physics 42(2), 837 (1971)

[16] P. Pusey, J. Phys. D: Appl. Phys. 9, 1399 (1976)

[17] D. Chowdhury, C.M. Sorensen, T. Taylor, J. Merklin, T. Lester, Appl. Opt. 23, 4149 (1984)

[18] D.R. Linde, ed., CRC Handbook of Chemistry and Physics (Taylor and Francis, Boca Raton, FL, 2007)

[19] J.B. Salmon, F. Destremaut et al., to be submitted (2007)

[20] W. Press, Numerical Recipies in C, Second Edition (Cambridge University Press, 2002) 